\title{
ARTÍCULO
}

\section{Comunidades de Aprendizaje como apoyo a un Sistema de Gestión del Conocimiento y la Innovación. Un estudio de Caso}

\author{
Gutiérrez, Ángel*, Lleras, Ernesto ${ }^{\circ}$, Diaz, Julia† \\ * Facultad Latinoamericana de Ciencias Sociales, Quito, Ecuador, \\ aagutierrez@flacso.edu.ec \\ ○ Facultad de Ingeniería, Universidad de los Andes, Bogotá, Colombia, \\ elleras@uniandes.edu.co \\ $†$ Facultad de Ingeniería, Universidad de los Andes, Bogotá, Colombia, \\ jh.diaz84@uniandes.edu.co
}

En el este artículo presentamos un estudio de caso donde se usa el enfoque de Comunidades de Aprendizaje (CA) para propiciar un espacio de innovación dentro de una organización. En el caso se busca fortalecer el Sistema de Gestión del Conocimiento y la Innovación (SGCI) de una empresa de servicios públicos, estableciendo relaciones de aprendizaje entre los trabajadores. Es estas relaciones de aprendizaje las personas asumen roles diferenciados (de mentores o aprendices) y pueden desarrollar capacidades que les permiten proponer nuevas prácticas a la empresa.

\section{Palabras clave:}

Comunidad de Aprendizaje, Capacidades Organizacionales, Aprendizaje Organizacional, Investigación Acción Participativa (IAP), Innovación.

\section{INTRODUCCIÓN}

Este artículo aborda la problemática de cómo propiciar espacios que incentiven la construcción de mundo ${ }^{1}$ dentro de la empresa, en función del desarrollo de capacidades organizacionales. En la literatura especializada se privilegian las investigaciones que buscan entender, clasificar,

\footnotetext{
${ }^{1}$ La construcción de mundo (Heidegger, 2015) hace referencia a la noción de Heidegger en construir, habitar, pensar, de construir hábitats que les permita a las personas desarrollar su autenticidad. Es una noción que será trabajada a lo largo del artículo.
} 
Gutiérrez, Lleras y Díaz

Comunidades de Aprendizaje como apoyo a un Sistema de Gestión del Conocimiento y la Innovación. Un estudio de Caso.

identificar y medir las capacidades para la innovación y su impacto en la competitividad (García et al., 2014). Son menos frecuentes los estudios sobre cómo incentivar el desarrollo de dichas capacidades en casos concreto, precisamente la temática que aborda el presente artículo. Aunque en la literatura especializada se reconoce la complejidad asociada al estudio de las capacidades, por ejemplo, en términos de las diferentes dimensiones asociadas a las capacidades de innovación (Yam et al., 2010; Wang et al., 2007; Guan et al., 2006), en dicha literatura se privilegia un enfoque asociado a los artefactos tecnológicos y su articulación en procesos de investigación y desarrollo $(\mathrm{I}+\mathrm{D})$.

En el artículo argumentamos que los actuales enfoques sobre innovación toman esa perspectiva artefactual en cuanto prima un determinismo tecnológico y económico en la forma de pensar la construcción de mundo. En el artículo se realiza un análisis crítico sobre las consecuencias y limitaciones de esta forma de pensar la innovación en el contexto colombiano, y se aboga por trascender esta la mirada del determinismo. Proponemos otro acercamiento y lo exponemos a través de un estudio de caso, a partir de otra manera de pensar la construcción de mundo. En esta mirada se reconoce la validez de entender las dinámicas de innovación en función de las capacidades necesarias para afrontar problemas. En este caso esas dinámicas se piensan en un marco afín al de la construcción social de la tecnología y no desde el determinismo tecnológico y económico. Lo que se resignifica frente a la comprensión habitual de la construcción social de la tecnología, es el privilegio del diálogo como centro de la construcción consciente de mundo y no las controversias sociales alrededor del uso los productos tecnológicos.

En el presente artículo ejemplificamos lo anterior partiendo de un estudio de caso, en el que una comunidad de aprendizaje busca constituirse en un espacio de cohesión creativa en el ámbito de la organización, y donde sus miembros puedan aprender a afrontar y solucionar las problemáticas en función de sus propias capacidades y talentos. El estudio de caso se desarrolla en la Empresa de Acueducto y Alcantarillado de Bogotá (EAAB-ESP), una entidad pública encargada de proveer el servicio de agua potable y alcantarillado en Bogotá, ciudad con aproximadamente ocho millones de habitantes.

El caso se centra en una de las comunidades de aprendizaje que la EAAB-ESP desarrolló para apoyar el diseño y la implementación su SGCI. En la EAAB-ESP el enfoque de CA se ha usado previamente, y en este caso, la comunidad se constituyó específicamente para diseñar e implementar mecanismos efectivos para que los trabajadores expertos compartieran su conocimiento con trabajadores de menos experiencia. La estrategia fue que la comunidad se estableciera como un espacio para desarrollar competencias pedagógicas en los trabajadores expertos, quienes compartirían su experticia al asumir el rol de mentores empresariales. Se hicieron talleres donde se desarrolló un proceso de reflexión-acción-reflexión a partir de las situaciones laborales vividas por los integrantes de la comunidad.

\section{ANTECEDENTES TEÓRICOS}

Partimos de la literatura especializada, donde se sugiere la posibilidad de que exista una relación positiva entre aprendizaje, efectividad e innovación en las organizaciones. La relación entre 
Gutiérrez, Lleras y Díaz

Comunidades de Aprendizaje como apoyo a un Sistema de Gestión del Conocimiento y la Innovación. Un estudio de Caso.

aprendizaje organizacional (AO) y la efectividad está bien establecida en sus elementos estructurales, a tal punto que el $\mathrm{AO}$ se considera fundamental para la viabilidad de las organizaciones en los entornos complejos actuales. Así, con relación al AO, los comportamientos organizacionales pueden ser estudiados desde una perspectiva sistémica (Senge, 1990; Checkland, 2000; Nair, 2001), donde la relación que se establece entre la identidad de una organización con su ambiente está en las raíces de la efectividad organizacional (Beer, 1981; Von Foerster, 2002).

Por otro lado, la articulación del AO y la efectividad organizacional con la innovación genera menos consenso en la literatura, aunque la mirada multidimensional muestra diferentes aspectos de esa articulación. En términos generales varios autores proponen que la relación entre aprendizaje y efectividad está mediada por la innovación: capacidades organizacionales $\rightarrow \mathrm{AO} \rightarrow$ innovación $\rightarrow$ desempeño (Giniunienea y Jurksiene, 2015). Esto aclara lo que anteriormente llamamos una relación positiva entre AO, innovación y efectividad, en cuanto hay innovación si la novedad que se introduce frente a los problemas, novedad que se propicia a través del aprendizaje, está alineada con los objetivos organizacionales.

Consideramos que la mirada anteriormente comentada soslaya aspectos asociados a la resolución de problemas pertinentes en nuestro país, como puede ser la manera como se gestionan las relaciones de poder entre los miembros de una organización, dado que lo que prevalecen son relaciones de dominación. Por esto, como un asunto de investigación en la acción, buscamos propiciar espacios laborales de aprendizaje que trasciendan los patrones de dominación mencionados. Una de las capacidades que se busca instalar en la organización es la gestión de las relaciones de poder, a través del enfoque de CA. Estos espacios son llamados "hábitats", y son ámbitos en los cuales los individuos desarrollan capacidades personales y organizacionales para la resolución de problemas, a la vez que reconfiguran los patrones de poder considerados nocivos frente a la resolución de problemas y para la convivencia. Un ejemplo de ello es promoviendo ámbitos de interacción donde el trabajador se convierta en agente en lugar de considerarse parte pasiva o en resistencia frente a las prácticas organizacionales.

De acuerdo con Garzón y Fisher (2008) el término AO aparece por primera vez en una publicación de Miller y Cangelloti (1965), quienes basados en la teoría de la contingencia y desde la concepción de una organización como organismo vivo, proponen un proceso de realimentación, ya sea para reforzar o cambiar comportamiento frente a la dinámica del ambiente. Así se establece un modelo de adaptación-aprendizaje para apoyar la viabilidad organizacional. En términos sociales, la propuesta ubica el conocimiento individual a disposición de la organización y es resignificado por diversos autores desde diferentes perspectivas, que abarcan las dimensiones individuales y sociales del aprendizaje, además de las estructurales comentadas organización-entorno como un proceso de retroalimentación.

Por ejemplo, en las propuestas de Polanyi (1967) hay dos tipos de conocimiento que pueden articularse, el tácito y el explícito, aunque no del todo diferenciados. El conocimiento tácito resulta de las experiencias y del aprender haciendo, una producción cultural internalizada y difícil de explicar a otros individuos. El conocimiento explícito o codificado es el saber externalizado y sistematizado en la esfera social, en cuanto puede ser fácilmente expresado en el habla. El primero es un conocimiento que se puede usar a nivel organizacional en cuanto es útil y práctico, y lo que propone Polanyi es que los individuos pueden aprender a expresarlo al desarrollar capacidades 
Gutiérrez, Lleras y Díaz

Comunidades de Aprendizaje como apoyo a un Sistema de Gestión del Conocimiento y la Innovación. Un estudio de Caso.

específicas, en una suerte de relaciones mentor-aprendiz, con ventajas para la organización en términos de "transferencia de conocimiento".

Argyris y Schon (1978) introducen la comprensión de dos niveles de aprendizaje, el nivel individual y el organizacional, así como el concepto de tipos de aprendizaje frente a los aspectos estructúrales del aprendizaje en el ámbito organización-entorno ya comentados. Así el primer tipo es de aprendizaje de ciclo simple y el segundo de ciclo doble, que despliega otras dimensiones del aprendizaje, como el "aprender a aprender". La concepción de Levitt y March (1988) relaciona el aprendizaje con la incorporación de inferencias de la historia en rutinas que guían el comportamiento, un aprendizaje a partir de las "lecciones aprendidas", en cuanto logran determinar el comportamiento organizacional.

\section{Las capacidades organizacionales}

Estos tres ejemplos, además de ser paradigmáticos, son perspectivas que fundan diferentes miradas sobre aspectos del AO y su relación con la efectividad, que además de la dimensión estructural abarca otros ámbitos, como el aprendizaje por experiencia directa, por intermedio de otros y por la historia. Además, muestran diversos aspectos de las capacidades que se buscan desarrollar frente al reto de afrontar los problemas organizacionales de manera efectiva. La influencia de perspectivas orgánicas-evolutivas sigue siendo fundamental en las discusiones sobre capacidades organizacionales, en particular los trabajos de Schumpeter sobre los procesos de innovación (Dávila 2013). En esta trayectoria se considera que el aprendizaje es un factor determinante si se busca el desarrollo de las capacidades necesarias para establecer las jerarquías de los comportamientos rutinarios y su alcance, con el fin de facilitar los procesos de adaptación organizacional.

Para Dávila (2013), entonces, la innovación se facilita si las rutinas son fiables y de amplio alcance, y en cuanto se establezca una observación del entorno, y una acción flexible y oportuna ante las situaciones imprevistas. En este sentido intervienen dos lógicas, la de la rutina y otra contraria a la de la rutina, pero articuladas en la práctica: la síntesis entre creatividad y rutina se posibilita en una estructura dialógica. Es una perspectiva coherente con la visión del mecanismo de adaptación desde la perspectiva cibernética de Beer (1981), donde la adaptación y viabilidad de las organizaciones depende de la tensión creativa entre la cohesión y la inteligencia organizacional. Desde el enfoque de CA se propone mirarlo como la paradoja cohesión-creatividad que se expresa en las relaciones de producción-creación.

Así, desde Dávila (2013), las capacidades organizacionales son fundamentalmente constructos sociales para solucionar los problemas de manera participativa, que evolucionan en el tiempo a través del aprendizaje. También, implican acción con propósito, en ámbitos de apertura, lo que permite la articulación de las lógicas diferentes comentadas, desde una reinterpretación de la estructura "tesis-antítesis y síntesis". Este aspecto ha sido abordado en el enfoque de CA frente a la paradoja de la cohesión-creativa, como un aspecto de la resolución de problemas complejos en un ámbito de reflexión-acción, que se mostrará más adelante.

Desde la perspectiva del AO, sin el desarrollo de capacidades organizacionales se dificulta que un grupo humano se organice empresarialmente en procesos de innovación que perduren en el tiempo. 
Gutiérrez, Lleras y Díaz

Comunidades de Aprendizaje como apoyo a un Sistema de Gestión del Conocimiento y la Innovación. Un estudio de Caso.

Como se ha observado, en la literatura se ha estudiado la relación entre capacidades organizacionales y la solución colectiva de los problemas de manera efectiva. Las capacidades organizacionales se construyen socialmente en ámbitos de aprendizaje (Dávila, 2012) y se pueden reconocer situadamente en cuanto se usan subsecuentemente para la solución efectiva de los problemas organizacionales.

Lo comentado anteriormente tiene implicaciones en términos de diseño organizacional, en particular para pensar en la posibilidad de desarrollar intencionalmente capacidades organizacionales. Por ejemplo, en el ámbito de la innovación subsecuente desde el paradigma schumpeteriano, o de manera más general, en el ámbito de la construcción de mundo. Más allá del paradigma estructural-evolutivo dominante, que se analizará críticamente frente a situaciones límites asociadas a la resolución efectiva de los problemas, compartimos el interés por fomentar el desarrollo de las capacidades organizacionales desde el horizonte planteado desde Dávila (2012), una capacidad estaría organizacionalmente instalada si se usa en varios momentos y diversas situaciones para resolver problemas de manera efectiva.

La noción de CA propone un enfoque en que se busca la construcción participativa de una matriz de relaciones sociales en la cual el aprendizaje y la construcción de mundo van de la mano. El aprendizaje se entiende como un proceso articulado por acciones con sentido (que nosotros llamamos prácticas) entre los participantes, en ciclos interminables de observación-reflexiónacción, inmersos en un espacio de diálogo, reconocimiento y afecto. Y la construcción de mundo se asocia con la noción de hábitat entorno a los procesos de trabajo, donde el sentido del trabajo se relaciona con la autoconstrucción personal, desde la idea de que el trabajo trasciende la dimensión productiva y está inextricablemente vinculado al significado de la vida (Heidegger, 2015).

Hay otras nociones como la de Comunidad de Práctica (CP), que también parte de la dimensión social y situada del conocimiento (Lave y Wenger 1991; Wenger, 1988), con relación a la experticia de los practicantes (Brown \& Duguid, 1991). Aunque es un enfoque que privilegia la reflexión sobre las prácticas frente a la abstracción teórica, entiende los procesos sociales del conocimiento desde una perspectiva instrumental y utilitaria y no como un asunto de construcción de sentido frente al mundo.

A nivel de los aspectos estructurales organización-entorno, en particular sobre la noción de viabilidad, se busca fundar el mecanismo de adaptación sobre tres elementos que dan fuerza a las relaciones que establecen los individuos: afecto, diálogo y reconocimiento. El desarrollo de capacidades para gestionar las relaciones de poder y de producción-creación permiten que la comunidad se haga cargo de la administración interna, y de la toma de decisiones asociada a la relación organización-entorno. Así, la paradoja cohesión-creación está en el centro de la adaptación organizacional.

\section{La Innovación como forma de entender la construcción de mundo}

El concepto de innovación desde sus inicios está relacionado con el AO, y privilegia las perspectivas económica y tecnológica para entender la construcción de mundo. Schumpeter (1934) 
Gutiérrez, Lleras y Díaz

Comunidades de Aprendizaje como apoyo a un Sistema de Gestión del Conocimiento y la Innovación. Un estudio de Caso.

propone cinco fuentes posibles de innovación: conquistar una nueva fuente de materia prima, introducir un nuevo método de producción, introducir al mercado un nuevo bien, abrir nuevos mercados e implantar nuevas estructuras en un mercado (por ejemplo, un monopolio). Algunos autores han enfatizado en el proceso de producción de la innovación (Gee, 1981; Pavón y Goodman, 1981; Pavón e Hidalgo, 1997), otros en sus aspectos creativos (Nelson y Winter, 1982; Perrin, 1995) y otras corrientes en la relación con la efectividad y la competitividad (Drucker, 1975; Porter, 1990; Leonard-Barton, 1995; Machado, 1997; Christensen, 2000).

Lo que se puede argumentar es que el paradigma de la innovación evolutiva comentado es una forma de entender la construcción de mundo desde el determinismo tecnológico en el marco de una propuesta económica. En este determinismo el cambio técnico articulado al modelo económico es el motor del cambio social y de la transformación del comportamiento de las colectividades; de ahí la pregunta de Heilbroner: “¿Son las máquinas el motor de la historia?” (Heilbroner, 1996). El cambio tecnológico se concibe como casi autónomo e independiente de cualquier consideración política o cultural, por lo que la gestión de la innovación es principalmente un asunto tecnológico. Por extensión la relación innovación y competitividad posiciona el avance técnico como "motor" de la competitividad, y con ello a las sociedades productoras de la tecnología, generalmente de países desarrollados, con una clara ventaja competitiva.

Las consecuencias sociales son diversas para los países o las sociedades no productoras de tecnología. Porque desde esta perspectiva la innovación no está relacionada con las habilidades de las personas para dar cuenta de las situaciones problemáticas, sino con las posibilidades de transferir tecnologías. Así, por ejemplo, "los planteamientos de Schumacher (1999), Max Neff (1991) o Kohr (1978), de la necesidad de buscar tecnologías apropiadas a las circunstancias locales, es descartada totalmente en estas visiones" (Lleras y Gutiérrez, 2008, p.9), en cuanto por definición son tecnologías que no aportan en cuanto no resuelven el dilema del desarrollo en términos de competitividad.

También hay perspectivas que consideran la innovación social como una de las alternativas al concepto de innovación tratado previamente, aunque hay menos consenso de lo que este concepto significa. Desde esta visión algunos autores privilegian a los productores y beneficiarios de la innovación, tal como a las comunidades que solucionan de forma novedosas sus problemas (Phills, Deiglmeier y Miller, 2008; Bacon, Faizullah, Mulgan y Woodcraft, 2008; Goldenberg, 2004). Otros enfatizan en los procesos que pueden modificar los sistemas sociales (Leadbeater, 2008; Nilsson, 2003; Young citado por Pearson, 2007). En otros casos la innovación social se relaciona principalmente a las organizaciones del tercer sector (Mulgan, 2006). Pero, en todo caso, se considera que el peso de la transformación a nivel global es soportado por la innovación técnica. Así, el cambio social depende de unos pocos actores, generalmente localizados en los países llamados desarrollados.

En el contexto de América Latina, esta perspectiva no permite entender las dinámicas sino desde conceptos como transferencia o maquila. Por esto, desde nuestra perspectiva, el punto central en debate es si toda innovación es intrínsecamente social, porque si ese es el caso, la dimensión social sería inherente a cualquier clase de innovación y se privilegiaría sobre lo artefactual. Y si es así, ignorar este hecho constituye un sesgo que no permite entender las posibilidades de innovar en los 
Gutiérrez, Lleras y Díaz

Comunidades de Aprendizaje como apoyo a un Sistema de Gestión del Conocimiento y la Innovación. Un estudio de Caso.

países o en sociedades no industrializadas. A nuestro parecer, lo que está en el fondo son distintas formas como se piensan las relaciones entre la tecnología y la sociedad frente a la construcción de mundo. La primera, ya comentada es la del determinismo tecnológico en el marco de una propuesta económica, y la segunda, que presentamos como contraste, es la de la construcción social de la tecnología.

\section{La construcción social de la tecnología como forma de entender la construcción de mundo}

Las corrientes que asumen que la innovación es inherentemente social buscan dar cuenta de las relaciones sociotécnicas que se articulan en los procesos sociales de innovación. La construcción social de la tecnología plantea que el cambio tecnológico y la innovación está determinado por controversias que establecen diversos actores sobre las tecnologías, a la luz de sus intereses (Bijker, Hughes y Pinch, 1987). Así, la innovación pasa a depender de procesos sociales que se realizan en espacios de intercambio, donde se privilegia el uso de los artefactos sobre su producción, y por tanto la toma de decisiones que realizan los usuarios frente a la actualidad del artefacto. En las dinámicas de tomas de decisiones frente al uso se van dando forma a las innovaciones sociotécnicas en cuanto los productores entienden las necesidades de uso.

Un ejemplo típico desarrollado por Bijker y Pinch (1987) es el de la bicicleta, donde su actualidad es producto de innovaciones asociadas a controversias sobre la funcionalidad del diseño en un momento específico. Es el caso de la bicicleta de rueda alta, un diseño altamente valorado por los hombres como símbolo de estatus y virilidad en la Inglaterra de finales del siglo XIX, pero que las mujeres y otros grupos consideraban inseguro. La controversia se cierra con la bicicleta segura, por medio de diferentes mecanismos sociales de clausura como por ejemplo las estrategias de publicidad realizadas por sus fabricantes.

Lo que la construcción social de la tecnología está privilegiando es la capacidad de los actores relevantes para problematizar su mundo, trascendiendo la visión instrumental del aprendizaje y la innovación. En nuestra opinión, es una visión coherente con la noción de AO y la solución de problemas mencionada arriba desde la perspectiva de Dávila. La razón es el paradigma asociado a la construcción social de la tecnología se puede entender como un fenómeno dialéctico de "tesis antítesis y síntesis", donde mecanismo de contradicción donde se expresa la "tesis-antítesis" toma la forma de controversia frente a la actualidad de una tecnología, y la síntesis se expresa en términos de mecanismos que clausuran la controversia (Gutiérrez, 2018).

Así, la innovación comienza a depender de procesos sociales de tensión creativa que toman lugar en interacciones y en espacios para la toma de decisiones, donde las partes involucradas dan forma a las innovaciones técnicas desde el uso. La innovación es entendida como un proceso participativo, aunque en controversia, en el marco del aprendizaje estratégico y la construcción de sentido; una controversia que los actores buscan cerrar a la luz de sus intereses.

En la construcción social de la tecnología la dinámica de las innovaciones no sigue una trayectoria lineal orientada por la optimización técnica, sino que depende del contexto social específico. Alrededor de un artefacto hay grupos sociales que interactúan en diferentes momentos, en una producción histórica que puede representarse más precisamente como red. Desde el pensamiento 
Gutiérrez, Lleras y Díaz

Comunidades de Aprendizaje como apoyo a un Sistema de Gestión del Conocimiento y la Innovación. Un estudio de Caso.

crítico el constructivismo es un poderoso instrumento para pensar el diseño, por cuanto permite descubrir la desigualdad social que se introduce en lo producido a la luz del cierre de la controversia, porque durante la controversia estaban presentes las relaciones de poder, que se ocultan una vez realizada la innovación. En la práctica, con el cierre de la controversia y la inserción efectiva de la innovación en el ámbito social, se construye es una nueva forma de habitar, en una suerte de continuidad en el cambio que deja visible únicamente lo funcional e instrumental (Lleras y Gutiérrez, 2008).

\section{El Enfoque de CA como una forma de entender la construcción de mundo}

Las problemáticas personales y sociales relacionadas con la dominación han sido un tema recurrente en los estudios sociales y en particular en los efectos que se generan en la conformación del sentido que damos a la acción (Freire, 1970; Bourdieu, 1997; Foucault, 2000). Los efectos se observan tanto en lo personal, por ejemplo, en la falta de responsabilidad y de alegría, en la imposibilidad de desarrollar las vocaciones. Los efectos en lo social están asociados fundamentalmente con la imposibilidad de construir mundo con los otros, desde la confianza y la solidaridad.

Los patrones de dominación se concretan en la vida organizacional (Lleras, 1997; Fals-Borda, 2001; Sicard, 1989; Freire, 1970), por ejemplo, en el paternalismo y el autoritarismo, y sus contrapartes, la falta de autonomía, la falta de participación, la resistencia al cambio o la infelicidad. Esto último cuando el trabajo no se percibe como ámbito de realización personal, sino como obligación. La generalidad de estos patrones de autoridad es lo que llamaremos patrones de dominación, que en situación extrema quedan inscritos como evidencia en el folclor, por ejemplo, en la música popular como en el caso colombiano. En este sentido, se busca trascender problemas asociados a diversos patrones de dominación identificados en las formas organizacionales que prevalecen en Colombia, y en generar en la sociedad latinoamericana.

Estas problemáticas también las hemos evidenciado empíricamente en diversos espacios empresariales colombianos, en los que hemos participado a través de procesos de investigaciónacción. En nuestra experiencia son problemáticas que no solo dificultan las dinámicas que buscan tratar de forma efectiva las situaciones problemáticas, sino que tienen efectos negativos en términos del desarrollo social y de convivencia dentro de las organizaciones. Para Lleras, en el marco de las organizaciones, dichas problemáticas están relacionados con la falta de autonomía y con la enajenación (Lleras, 1997), ambas situaciones asociadas con la acción técnica.

En cuanto a la falta de autonomía, hemos hallado comportamientos paternalistas que trascienden del entorno familiar hacia la empresa, los cuales dificultan que las personas articulen coherentemente el sentido de la vida con el sentido del trabajo. Son comportamientos que a su vez afecta negativamente el desempeño de las organizaciones. La razón es que la persona autónoma es responsable, se hace cargo de su vida, tiene la capacidad de llevar a cabo su trabajo a partir de la comprensión que tiene del mismo, así como de reflexionar colectivamente sobre las formas organizacionales de las que participa y de proponer cambios a situaciones problemáticas que se presenten. En cuanto a la enajenación, ésta se deriva del uso sin sentido de constructos tecnológicos 
Gutiérrez, Lleras y Díaz

Comunidades de Aprendizaje como apoyo a un Sistema de Gestión del Conocimiento y la Innovación. Un estudio de Caso.

impuestos, lo que no le permite a la persona pensar en formas efectivas de articular la tecnología al trabajo.

Frente a la idea de la innovación subsecuente, hay una cuestión crítica en cuanto se soporta en capacidades instaladas en la organización más allá de los individuos. Pero en la práctica, lo que Lleras (2006) ha visto en organizaciones con problemas de autonomía y enajenación es que sin desarrollar ciertas las capacidades personales es muy difícil la instalación de las capacidades organizacionales (Lleras, 2006). El enfoque de CA busca desarrollar capacidades personales y organizacionales. Y aunque la relación entre ambos tipos de capacidades aún no es del todo clara, esta propiedad emergente sucede como se verá más tarde. En este sentido, la apuesta en CA trasciende la noción de influencia funcional lineal en la dirección de una influencia circular persona-comunidad-organización-persona.

En CA, se busca desarrollar la capacidad de observar relaciones, lo que perite la reflexión sobre las prácticas organizacionales con relación a la vida personal y colectiva. El ser humano continuamente evalúa la efectividad de la acción a la luz del sentido (Heidegger, 1996; Dreyfus, 1996). Así, los mismos intereses instrumentales o funcionales de la vida organizacional son subsidiarios de la construcción del sentido personal y colectivo. "Dar sentido" implica dar dirección a la acción, y la acción con sentido la entendemos como práctica. Esto nos permite probar nuevas formas de actuar y evaluar sentidos.

Así se hace posible construir mundo con los otros, ya sea para conservar lo instituido o para transformarlo. En este último caso, el ámbito de la innovación, el espacio emancipatorio se posibilita al establecer relaciones dialógicas que se encaminen hacia una construcción consciente del mundo, y es en este caso donde emerge la comunidad de aprendizaje. Así, las controversias sociales no son los únicos mecanismos de transformación social, y en muchos casos, hay otros espacios más relevantes, como el que se privilegia en el caso de las $\mathrm{CA}$, el diálogo como fundamento de la comprensión. En este sentido, la construcción de mundo presente en el enfoque de CA, aunque comparte aspectos comentados con relación a la construcción social de la tecnología, plantea un proceso no basado en la controversia, sino en el diálogo.

\section{METODOLOGÍA Y EL ESTUDIO DE CASO}

\section{Antecedentes del Proyecto}

La EAAB-ESP es una empresa de ingeniería con más de 130 años de existencia, reconocida como una autoridad a nivel nacional e internacional en la prestación de servicios de acueducto y alcantarillado. En sus políticas de personal y en su planeación estratégica ha habido un interés por contar con un SGCI basado en la experiencia que ganan sus trabajadores durante su tránsito por la empresa. Este interés se tradujo en propuestas de proyectos que fueron articuladas al concepto de innovación, entendido como una forma de poder aprovechar el conocimiento de sus trabajadores para resolver con prontitud los problemas de la empresa, principalmente los relacionados con la prestación de sus servicios. 
Gutiérrez, Lleras y Díaz

Comunidades de Aprendizaje como apoyo a un Sistema de Gestión del Conocimiento y la Innovación. Un estudio de Caso.

Uno de los problemas reconocidos por algunos directivos era la pérdida de conocimientos prácticos cuando los expertos terminaban la vida laboral. Los "expertos se iban y se llevaban su conocimiento". A la luz de esta problemática en el 2009 se propone un proyecto para que los trabajadores reconocidos como expertos apoyaran el desarrollo de las competencias de los trabajadores con menos experiencia. Fue un proyecto liderado por dos áreas de la empresa, las Gerencias de Tecnología y de Gestión Humana, a partir de un "proyecto piloto". Este proyecto sería desarrollado con el apoyo de las relaciones universidad-empresa que la EAAB-ESP había históricamente desarrollado. En este contexto se propuso una alianza con la Universidad de los Andes como facilitador del proyecto, el cual se desarrollaría con el enfoque de CA, usado por la organización previamente en el desarrollo de otros proyectos, por ejemplo, en el diseño del SGCI.

Con base en lo anterior se acordaron los entregables que se esperaban obtener del prototipo, en particular la propuesta de un modelo pedagógico que soportara las relaciones de aprendizaje entre mentores y aprendices. En este marco contextual, la EAAB-ESP inicia sus trabajos con una comunidad de aprendizaje formada por personas reconocidos como expertos en los niveles operativo y técnico, con el fin de construir participativamente los productos acordados. El diseño estaría acompañado de sugerencias sobre su implementación. Implicaba desarrollar en las personas expertas un conjunto de capacidades que les permitiera entrenar a los funcionarios que se vinculaban a la empresa, y conformar una red de apoyo para que todo funcionario que quisiera recurrir a los expertos pudiera hacerlo, a partir de problemas laborales concretos. Los trabajos inician bajo el nombre Proyecto Comunidad de aprendizaje para desarrollar competencias pedagógicas en mentores expertos.

\section{EI trabajo de la comunidad}

La problemática que primero abordó la CA de mentores tenía que ver con la falta de una inducción y entrenamiento adecuado y sus efectos en la efectividad organizacional. Mejorar la situación requería aplicar de una estrategia en la cual el conocimiento acumulado mediante la experiencia fuera compartido en las prácticas operativas, de tal forma que se "aprendiera haciendo". Para tal fin los tutores expertos debían afinar su capacidad de observación y apoyar al aprendiz en el desarrollo de su propia capacidad de observación. Esto sería apoyado con herramientas como se mostrará más adelante.

El enfoque tecnológico se entendió como un apoyo a las prácticas laborales. Por ejemplo, los medios TIC eran útiles de apoyo en las prácticas operativas, así como todos los instrumentos técnicos que conforman la "caja de herramientas" que usa el trabajador. Un objeto técnico debe ser pertinente para el trabajo y los problemas sobre su uso pasan por la reflexión de los trabajadores, a tal punto que pueden rediseñados. Así el motor de la innovación es el "hacer las cosas bien", lo que finalmente se traduce en "capital social e intelectual de la empresa", traducido en elementos como, por ejemplo, buenas prácticas laborales y patentes.

A nivel organizacional se fue fortaleciendo la idea de posicionar los asuntos ambientales y de responsabilidad social empresarial en la ingeniería de la empresa, debido a que la primacía del enfoque tecnológico en las actividades misionales de la organización impedía mirar otros aspectos 
Gutiérrez, Lleras y Díaz

Comunidades de Aprendizaje como apoyo a un Sistema de Gestión del Conocimiento y la Innovación. Un estudio de Caso.

fundamentales. Asimismo, se acordó que el enfoque de CA seleccionado permitiera la construcción colectiva de la articulación de las temáticas con la tecnología, y desde acá, se generaran los lineamientos para la gestión del conocimiento de la Empresa.

Así se fueron configurando espacios de aprendizaje donde las personas, a través de la reflexión sobre sus prácticas en las dimensiones técnicas, operativas y de gestión, capitalizan la experiencia, generan innovación en sus prácticas, y proponen nuevas prácticas en pro del desarrollo humano y de la sostenibilidad empresarial. La construcción colectiva implicaría promover relaciones de confianza, así como fortalecer la autonomía responsable y el trabajo en equipo, lo cual, como se mostrará a continuación, estaría mediado por la estrategia del trabajador como empresario de su puesto de trabajo.

\section{Elementos teórico-metodológicos del enfoque de CA en acción}

Metodológicamente, para la conformación de la comunidad, se les propuso a los expertos participantes un enfoque basado en el diálogo. Se buscaba desarrollar capacidades pedagógicas en los mentores y dar cumplimiento a lo pactado con las directivas. Las reflexiones en la acción partieron de que cada individuo en su puesto de trabajo podía observar el proceso de mentoría con los trabajadores que estaba capacitando, de los niveles técnico y operativo. Las reflexiones sobre el sentido de la vida y la relación de dicho sentido con el trabajo, así como las reflexiones sobre las prácticas de mentoría, llevaron a concebir las relaciones mentor-aprendiz como un asunto de crecimiento personal, que aportaba también a la organización. Lo que articula el sentido de la vida con el sentido del trabajo es el servicio: "se crece como persona y se aporta a la organización en cuanto se sirve".

Como se puede notar, para construir el espacio de diálogo se buscó articular la percepción y reflexión de los individuos sobre sus propias prácticas de mentoría, las cuales eran expresadas y socializadas en espacios de encuentro comunitario. La reflexión colectiva se inserta en una dinámica donde se aprende a compartir las experiencias, en cuanto se comienza a observar las prácticas, generalmente vistas en su dimensión técnica y operativa, en el marco de la seguridad personal. En el caso de las CA dichas prácticas se observaron en un marco más amplio, en cuanto se desarrollan capacidades para observar y gerenciar tres tipos de relacionales: las relaciones de comunicación, de producción-creación y de las relaciones de poder. Aquí emergen nuevos espacios de reflexión, donde las personas pueden generar innovación técnica y proponer nuevas prácticas que favorezcan el crecimiento humano. Esto se puede contrastar con las prácticas en una $\mathrm{CP}$, donde las personas privilegian la mejora como un asunto de desarrollo técnico especializado.

Acorde al enfoque de CA, nos propusimos desarrollar capacidades personales y organizacionales para gestionar los tres tipos de relaciones comentadas. Se propusieron herramientas de observación personales, como las bitácoras para observar las prácticas de mentoría, y otras organizacionales, como los cuadros enriquecidos, dibujos para observar y estructurar problemáticas en la organización (Checkland, 1981), y las "lecciones aprendidas". Fueron herramientas útiles para analizar los procesos de trabajo y las prácticas de aprendizaje implicadas en estos procesos, en la labor de mentoría. Las herramientas fueron útiles, apoyaron a que la CA se diera cuenta de los 
Gutiérrez, Lleras y Díaz

Comunidades de Aprendizaje como apoyo a un Sistema de Gestión del Conocimiento y la Innovación. Un estudio de Caso.

errores que se pueden llegar a cometer si se cae "en la trampa de la rutina", entendida como aquellos comportamientos asociados a una "visión estrecha", no acorde a las dinámicas cambiantes del entorno, y por tanto una visión impertinente para dar cuenta de los problemas que buscan solucionarse.

Lo que se observa es que "la trampa de la rutina" está íntimamente relacionada con la falta de desarrollar capacidades que permitan observar las múltiples dimensiones relaciones pertinentes a las situaciones específicas, que deben ser gestionadas. Por esto, la propuesta implicó que el mentor debía desarrollar la capacidad de observar las actividades laborales en el marco relacional de apertura comentado, así como de mostrar los aspectos del trabajo con la intención de compartir experiencias de gestión. Esto ayuda a construir el escenario que permite una comunicación coherente con las personas que están bajo su responsabilidad, una construcción social mediada por la CA.

La reflexión sobre los procesos de comunicación, con relación a los patrones de dominación existentes, llevó a que la CA planteara una propuesta de gestión de los procesos comunicativos alternativa, basada en el aprendizaje más que en las prácticas tradicionales de control, órdenes y memorandos. Las reflexiones sobre el poder y su relación con las formas de comunicación se inscribieron en el desarrollo de una pedagogía constructivista y activa, la cual exige al mentor estar en actitud receptiva, destacando los aportes de los demás en la construcción de aprendizaje.

Se acordó que el aprendizaje colectivo debía permitir construir la confianza requerida, a partir del desarrollo de la autonomía del trabajador, bajo la estrategia de ser gerente de su puesto de trabajo. Aquí está presente un símil del dilema ya tratado por autores como Bordieu (1997), entre autonomía y compromiso, en el marco de campos especializados y jerárquicos, que define posiciones en los individuos, muy en la línea de las relaciones y posiciones asociadas a los roles empresariales. La estrategia del trabajador como gerente de su puesto de trabajo busca precisamente "aplanar" la organización trascendiendo la idea de especialización: todos son gerentes de su puesto de trabajo.

Pero dicha estrategia implica el desarrollo de las capacidades organizacionales de gestión de las relaciones comentadas. Así, el dilema autonomía y compromiso se resuelve en el enfoque de CA en términos de capacidades relacionales que permiten hacerse cargo de la propia vida - auto gestión de la vida personal, a la vez que soportan el desarrollo de las capacidades organizacionales de gestión de las tres relaciones comentadas. Por esto, las personas como empresarios de su puesto de trabajo se encargaron de documentar las experiencias, y de compartirlas en los talleres con otros mentores en la comunidad de mentores expertos, constituyéndose así un espacio de aprendizaje para la gestión de dichas relaciones.

El desarrollo de las capacidades para gestionar el poder se centró es establecer procesos de auto reconocimiento y de reconocimiento del otro. Nosotros descubrimos que tanto para la compañía como para quienes fueron reconocidos como mentores expertos, el proceso de reconocimiento contribuyó al desarrollo de la autonomía y de un sentido de orgullo por el trabajo bien hecho. Se inició por generar nuevas prácticas de reconocimiento dentro de la $\mathrm{CA}$. El proceso se basó en el mérito y no en elementos culturales tales como el favoritismo, u otras prácticas indeseables. 


\section{Los resultados del prototipo}

De estas reflexiones emergieron los lineamientos pedagógicos para presentar a la Empresa un producto tangible del trabajo de la comunidad, y con ello dar cumplimiento a su relación de servicio. Los lineamientos pedagógicos se propusieron como guías que promovían la reflexión pedagógica personal y colectiva, desde las prácticas que se llevan a cabo en la EAAB-ESP. Así se generaron espacios para el intercambio de experiencias, que buscaban orientar el desarrollo de las capacidades relacionales comentadas. Era una propuesta para el fomento del SGCI a través de la reflexión y la transformación de las relaciones que se establecieron entre mentores y aprendices, dentro del contexto de una comunidad de trabajadores.

La propuesta implicaba que a través de los procesos de mentoría se incentivara en la empresa la construcción de un nuevo hábitat, donde las personas desarrolles su individualidad a través del incentivo que produce el llevar a buen término las relaciones de servicio, en términos de calidad, pertinencia, efectividad y estética. Se propuso que la misma comunidad de aprendizaje se desarrollara a partir de tres $\mathrm{CP}$, en cuanto las prácticas técnicas y operativas implicaban conocimientos especializados específicos. Estas fueron la comunidad de Acueducto, la de Alcantarillado y la Comunidad SISO que observaría las buenas prácticas con relación a la seguridad.

Los participantes de la comunidad de mentores llegaron a acuerdos y compromisos sobre las condiciones que permiten construir un espacio que posibilite el diálogo, basado en lo que han aprendido en las diferentes CA desarrolladas en el pasado, la actual de mentores, y de la perspectiva de la construcción futura de espacios de diálogo, de tal forma que, mientras se insertaban la prácticas de mentoría en los diferentes dominios operativos y técnicos, se fuera construyendo el hábitat organizacional. Se construyó un modelo en que se articularon los principales elementos comentados anteriormente. La relación mentor-aprendiz estaba mediada por conceptos como el diálogo, la comunidad de aprendizaje y el reconocimiento (Figura 1). 


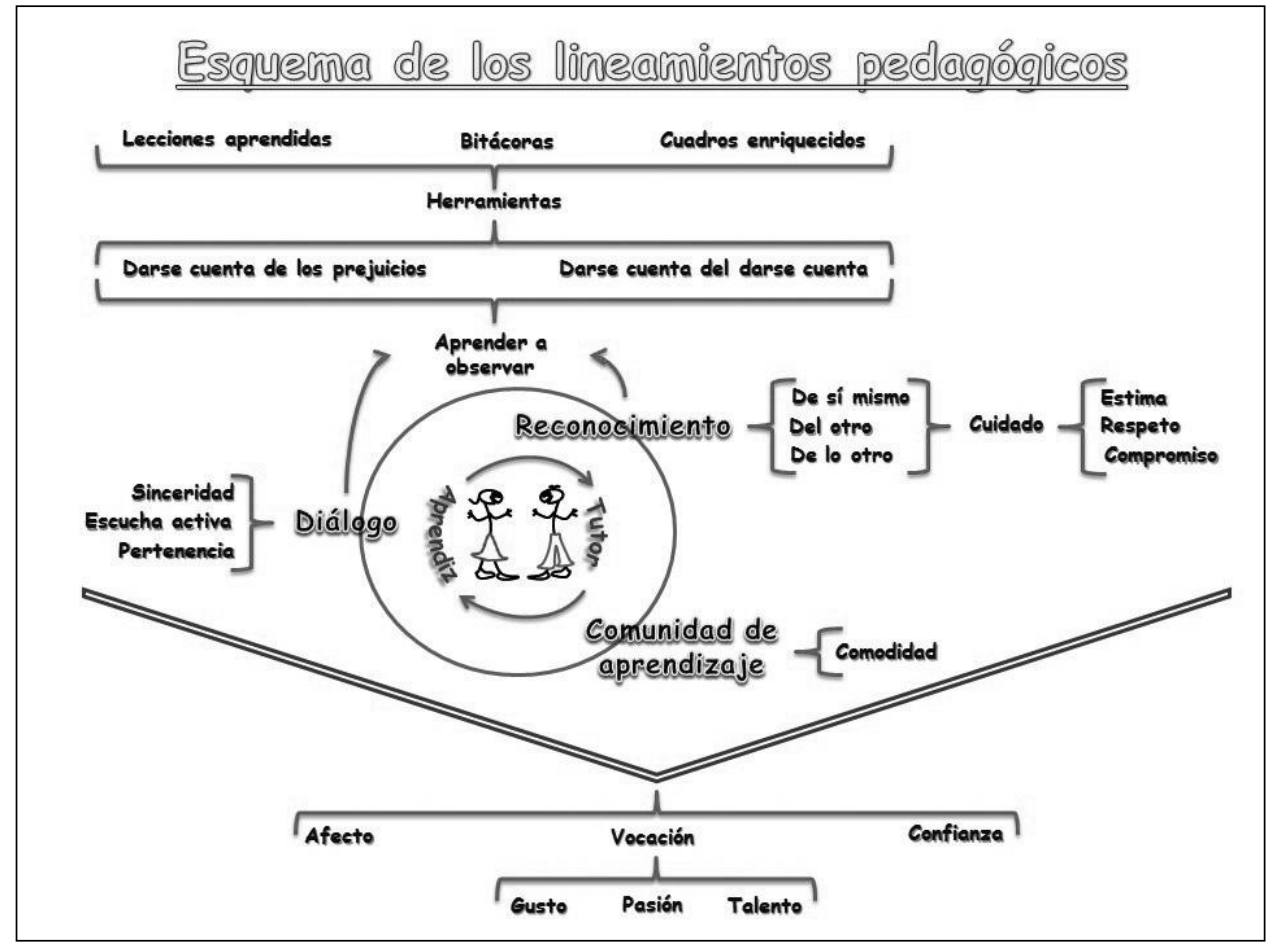

Figura 1. Esquema de lineamientos pedagógicos propuesto por la comunidad.

Así mismo, la propuesta implicaba elementos mínimos necesarios que se beberían tener en cuenta para "aplanar la organización" desde la noción del trabajador como gerente de su puesto de trabajo. Este sería un aspecto central de construir un actuar autónomo y basado en la confianza y se pensaron movilizar desde la conformación de tres roles asociado a las mentorías: el primero, el de aprendiz, en estado de descubrimiento de su vocación por medio del trabajo. El segundo, el de mentor, que acompaña los procesos de aprendizaje. Y el tercero, el de jefe como coordinador, que reconoce la experiencia del mentor e incentiva el aprendizaje del aprendiz.

Así, la comunidad planteó un modelo donde se argumentaba la importancia de que un trabajador estuviera en continuo aprendizaje a la luz de sus prácticas, en el ámbito del crecimiento personal anteriormente expuesto. Lo que se formaría era en un hábitat, donde el trabajador articulaba con sentido la pertenencia a la empresa, y la responsabilidad social y ecológica de su actuar, más allá de la empresa. Así, la comunidad concluyó que el "fin último" de su trabajo tiene que ver con el servicio y cuidado del medio ambiente. Un elemento fundamental del proceso de aprendizaje, porque el trabajo adquiere una dimensión de solidaridad y compromiso con el otro, con los otros y con la ecología del planeta.

\section{CONCLUSIÓN}


Gutiérrez, Lleras y Díaz

Comunidades de Aprendizaje como apoyo a un Sistema de Gestión del Conocimiento y la Innovación. Un estudio de Caso.

Los resultados apuntan a que el ejercicio permitió articular una propuesta innovadora para la estrategia de formación. Esto se logó desde el reconocimiento del valor de las prácticas y de la experticia de los trabajadores. Así, el reconocimiento era uno de los ejes de la relación mentoraprendiz, en el marco de tres CP que alimentan las reflexiones de la comunidad desde la operación. Estos elementos se configuraron a partir de trabajo previos con el mismo enfoque, lo que permitió una respuesta ágil a la problemática planteada, un trabajo que aprovechó las capacidades instaladas en la organización, que en el caso concreto duró aproximadamente dos meses.

En el experimento se observa que los trabajadores estaban interesados en transformar algunas relaciones sociales a partir de observar críticamente el diseño organizacional existente. Era una necesidad sentida, la de superar algunos patrones de dominación asociados a la jerarquía, que partía de entender la empresa desde la imagen de la máquina. Se buscó que la EAAB-ESP se dirigiera hacia hábitats de personas reflexivas, autónomas, conscientes de su continuo proceso de aprendizaje en el puesto de trabajo, El concepto del reconocimiento (Lévinas, 1999) fue resignificado por los trabajadores y mostró su utilidad en el desarrollo de las capacidades requeridas para gestionar las relaciones de poder.

Cuando el individuo asume su responsabilidad en el trabajo, también descubre y desarrolla su capacidad para gestionar las relaciones de poder, así como las de gestión. Esto es, desarrolla prácticas que le permiten lidiar con la paradoja cohesión-creación, en un ámbito que le permite observase como individuo en colectividad. Las capacidades personales y organizacionales se desarrollan y refuerzan a partir de la reflexión sobre la acción en el puesto de trabajo, reflexiones que se socializan en el ámbito de la comunidad de aprendizaje. Esta capacidad de reflexión en la acción permite que la dimensión técnica se piense desde un marco que supera la visión determinista. La caja de herramientas que se ha venido usando en el trabajo se resignifica. Se pasa de un entendimiento del conjunto de artefactos como mecánicos y de uso repetitivo, a entenderlos como un elemento crucial para el desarrollo de la excelencia laboral

El diálogo se comienza a revelar como una experiencia muy valiosa no solo para trabajar las relaciones de poder, sino desde el punto de vista de la motivación. Creemos también que comienza a emerger una comprensión de la enorme capacidad que se puede desarrollar al emplear el diálogo para propiciar el trabajo en equipo. Porque el diálogo incentiva un estilo de trabajo que, junto con la sinceridad y el reconocimiento de los talentos individuales, logran mayor sinergia y beneficia el desarrollo de la comunidad en términos de convivencia.

\section{REFERENCIAS}

Argyris C, Schön D. (1978). Organization Learning II. Theory, Method and Practice. Reading, Massachusetts: Addison-Wesley.

Bacon N, Faizullah N, Mulgan G, Woodcraft S. (2008). Transformers: how local areas innovate to address changing social needs. London: NESTA.

Beer S. (1981). Brain of the firm. New York: John Wiley. 
Gutiérrez, Lleras y Díaz

Comunidades de Aprendizaje como apoyo a un Sistema de Gestión del Conocimiento y la Innovación. Un estudio de Caso.

Bijker W, Hughes T, Pinch T. (1987). The Social Construction of Technological Systems: New Directions in the Sociology and History of Technology. Massachusetts: The MIT Press.

Bordieu P. (1997). Razones prácticas sobre la teoría de la acción. Barcelona: Anagrama.

Brown J, Seely P, Duguid P. (1991). Organizational Learning and Communities-of-Practice: Toward a Unified View of Working, Learning, and Innovation. Organization Science, 2(1), Special Issue: Organizational Learning: Papers in Honor of (and by) James G. March: 40-57.

Checkland P. (1981). Systems Thinking, Systems Practice. Chichester: John Wiley.

Checkland P. (2000). Soft systems methodology: a thirty-year retrospective. Systems Research and Behavioral Science, 17(1), S11-S58.

Christensen C. (1997). The Innovator's Dilemma. EEUU: Collins.

Dávila J. (2012). La doble dimensión de una capacidad organizacional: evidencias de una organización sin ánimo de lucro que compite en el mercado. Cuadernos de Administración, 25(44), 11-37.

Dávila J. (2013). Capacidades organizacionales: dinámicas por naturaleza. Cuadernos de Administración, 26(47), 11-33.

Dreyfus H. (1996). Ser-en-el-Mundo. Santiago de Chile: Editorial Cuatro Vientos.

Drucker P. (1975). La gerencia: tareas, responsabilidades y prácticas. Buenos Aires: El Ateneo.

Echeverría R. (2002). Ontología del lenguaje. Santiago de Chile: Dolmen.

Fals-Borda O. (2001). Participatory (Action) research in social theory: origins and challenges. In Handbook of action research Participative enquiry and practice. Reason P, Bradbury H (Eds). London: Sage, 27-37.

Freire P. (1970). Pedagogía del Oprimido. Siglo XXI: Bogotá.

Fromm E. (1966). Marx y su concepto del hombre. Ciudad de México: Editorial Fondo de Cultura Económica.

Foucault M. (2000). Vigilar y Castigar. Nacimiento de la prisión. México: Editorial Siglo Veintiuno.

García, O., Quintero, J. y Arias, J. (2014). Capacidades de innovación, desempeño innovador y desempeño organizacional en empresas del sector servicios. Cuadernos de Administración, 27 (49), 87-108.

Garzón M, Fischer A. (2009). El aprendizaje organizacional en República Dominicana y Colombia. Pensamiento \& Gestión, (26), 239-278.

Gee S. (1981). Technology transfer, innovation \& international competitiveness. New York: Wiley \& Sons.

Giniuniene J., Jurksiene, L. (2015). Dynamic Capabilities, Innovation and Organizational Learning: Interrelations and Impact on Firm Performance. Procedia - Social and Behavioral Sciences 213 (2015) 985-991. 
Gutiérrez, Lleras y Díaz

Comunidades de Aprendizaje como apoyo a un Sistema de Gestión del Conocimiento y la Innovación. Un estudio de Caso.

Goldenberg M. (2004). Social Innovation in Canada: How the non-profit sector serves Canadians... and how it can serve them better. Ottawa: Canadian Policy Research Networks.

Guan, J., Yam, R., Mok, C., and Ma, N. (2006). A study of the relationship between competitiveness and technological innovation capability based on DEA models. European Journal of Operational Research, 170, 971-986.

Gutiérrez, A. (2018). Transformación de la política CTI en Colombia, en el 'ámbito de la Ley 1286 de 2009. Una perspectiva de redes de política. Facultad Latinoamericana de Ciencias Sociales: Quito: FLACSO-Ec.

Gutiérrez, A. Díaz, J. (2010). Herramientas organizacionales en la construcción de una comunidad de aprendizaje. Memorias ESOCITE 2010: Buenos Aires, Argentina.

Heidegger, M. (2015). Construir Habitar Pensar (Bauen Wohnen Denken), trad. por Jesús Adrián Escudero y Arturo Leyte. Barcelona: La Oficina.

Heidegger, M. (1996). Being and Time, trad. por Joan Stambaugh. Albany. New York: State University of New York Press.

Heilbroner R. (1996). ¿Son las máquinas el motor de la historia? In Historia y determinismo tecnológico, Merrit S, Marx L (Eds). Madrid: Alianza, 69-82.

Lave J, Wenger E. (1991). Situated learning: legitimate peripheral participation. New York: Cambridge University Press.

Leadbeater C. (1997). The rise of the social entrepreneur. London: Demos.

Leavitt B, March, J. (1998). Organizational Learning. Annual Review of Sociology, 319-340.

Leonard-Barton D. (1995). Wellsprings of Knowledge: Building and Sustaining the Sources of Innovation. Boston: Harvard Business School Press.

Lévinas E. (1999). Alterity and Transcendence. Columbia: University Press.

Lleras E. (2006). Comunidades de Aprendizaje como oportunidades emancipatorias. Memorias ESOCITE 2006. Bogotá.

Lleras E. (2002). Las comunidades de aprendizaje como ámbitos de construcción de mundo. In El Manual de iniciación pedagógica al pensamiento complejo, Velilla M (Comp). Quito: ICFES- UNESCO, 165-173.

Lleras E. (1997). Exposición sobre la Metodología TESO y un caso de estudio. In: Estrategia, Competitividad e Informática, Galviz Á, Espinosa Á (Comps.). Bogotá: Ediciones Uniandes, 171186.

Lleras E, Gonzáles G. (1991). El Trabajo como Dador del Sentido. Sistémica, 2(1), 17-26.

Lleras E, Gutiérrez A. (2008). La relación entre Tecnología y Sociedad vista como diseño. Río de Janeiro: Memorias ESOCITE 2008.

Machado F. (1997). Gestión tecnológica para un salto en el desarrollo industrial. Madrid: CDTICSIC. 
Gutiérrez, Lleras y Díaz

Comunidades de Aprendizaje como apoyo a un Sistema de Gestión del Conocimiento y la Innovación. Un estudio de Caso.

Miller I, Cangelloti C. (1965). Momentum and Revolution, organization adaptation. Academy of Management Journal, 23(4), 5591-614.

Mulgan G. (2006). The Process of Social Innovation. Innovations: Technology, Governance, Globalization, (1)2, 145-162.

Nair K. (2001). Adaptation to creation: progress of organizational learning and increasing complexity of learning systems. Systems Research and Behavioral Science, 18, 505-521.

Nelson R, Winter S. (1982). An evolutionary theory of economic change. New York: Harvard University Press.

Nilsson W. (2003). Social Innovation: An Exploration of the Literature. McGill Dupont Social Innovation Initiative. Montreal: McGill University.

Parker J. (2000). Let Your Life Speak. Listening for the Voice of Vocation. San Francisco: JosseyBass Inc.

Pavón J, Goodman R. (1981). Proyecto MODELTEC. La planificación del desarrollo tecnológico. Madrid: CDTI-CSIC.

Pavón J, Hidalgo A. (1997). Gestión e innovación: un enfoque estratégico. Madrid: Pirámide.

Perrin B. (1995). Evaluation and future directions for the Job Accommodation Network (JAN) in Canada. Final Report. Employment Policies and Operations, HRDC.

Pearson K. (2007). Accelerating our impact: Philanthropy, innovation and social change. Montreal: The J. W. McConnell Family Foundation.

Phills J, Deiglmeier A, Miller, D. (2008). Rediscovering Social Innovation. Stanford Social Innovation Review. 7, 34-43.

Piaget J. (1971). Psicología de la Inteligencia. Buenos Aires: Siglo XX.

Polanyi M. (1967). The tacit dimension. Gloucester: Peter Smith.

Porter M. (1990). The Competitive Advantage of Nations. New York: Free Press.

Robinson K, Aronica L. (2014). Finding Your Element. How to Discover Your Talents and Passions and Transform Your Life. New York: Penguin Books.

Searle J. (1969). Speech acts: an essay in the philosophy of language. London: Cambride University Press.

Senge P. (1990). The Fifth Discipline: The Art and Practice of the Learning Organization. New York: Doubleday.

Schumpeter J. (1934). The theory of economic development. New York: McGraw-Hill.

Sicard R. (1989). Teoría A, Concepción empresarial para el colombiano. In Dirección Ejecutiva Bogotá, 20(238), 161-188.

Von Foerster V. (2002). Sistémica Elemental desde un punto de vista superior. Medellín: Fondo Editorial Universidad EAFIT. 
Gutiérrez, Lleras y Díaz

Comunidades de Aprendizaje como apoyo a un Sistema de Gestión del Conocimiento y la Innovación. Un estudio de Caso.

Vygotsky L. (1978). Mind in Society: The development of higher psychological processes. Cambridge, MA: Harvard University Press.

Wang, C.; Lu, I. y Chen, C. (2007). Evaluating firm technological innovation capability under uncertainty. Technovation, 28 (1), 349-363.

Wenger E. (1998). Communities of practice: learning, meaning, and identity. Cambridge: Cambridge University Press.

Yam, R. C. M.; Lo, W.; Tang, E. P. Y. y Law, A. K. W. (2010). Technological innovation capabilities and firm performance. World Academy of Science, Engineering and Technology, 42, 1009-1017. 